\title{
An electrochemical sensor based on NiAl-layered double/graphene oxide hydroxide for detection of Vitamin B6
}

\author{
Chaolin Wang ${ }^{1}$, Yaping Ding ${ }^{1}, \mathrm{Li} \mathrm{Li}^{1}$, Qianwen Mei ${ }^{1}$, Anqing Wang ${ }^{1}$, Dingding Duan ${ }^{1}$, Hongyan Zeng ${ }^{1}$ \\ ${ }^{1}$ Department of Chemistry, Shanghai University, Shangda Road, Baoshan District, Shanghai 200444, \\ P.R.C \\ Corresponding e-mail address: wdingyp@sina.com, lilidu@shu.edu.cn
}

\begin{abstract}
:
Vitamin B6 (VB6) is an important part of vitamin B, involved in amino acid metabolism, carbohydrate and fat metabolism and hemoglobin formation. NiAl-layered double hydroxide (NiAl-LDH) and graphene oxide (GO) were complexed by hydrothermal method to form NiAl-LDH/GO composite. The NiAl-LDH/GO modified composite electrode was characterized by XRD, SEM and EIS, and presents a good electrocatalytic performance towards the oxidation of VB6. Under optimal experimental conditions, the linear response range for VB6 is $0.08 \mu \mathrm{M}$ to $500 \mu \mathrm{M}$, with the low limit detections of $0.027 \mu \mathrm{M}$. In addition, the electrode shows a good stability and high sensitivity. The new modified electrode can be used as electrochemical sensors.
\end{abstract}

Key words: NiAl-layered double hydroxide, graphene oxide, Vitamin B6, electrochemical sensor

\section{Introduction}

$B$ vitamins are essential nutrients for the normal metabolism of human[1]. VB6 is an important biomolecule that is widely involved in the body's metabolic, physiological and developmental processes[2]. VB6 plays an important protective role in the human body. It inhibits all kinds of cancer cells and their derivatives, reduce the incidence of cancer, simultaneously[3]. Therefore, VB6 can be used as a specific transport carrier to carry cells in human body[4]. However, due to the sluggish electron transfer processes and high over-potential, the voltammetric signal is not significant on bare glassy carbon electrode (GCE). Enormous effort have been put into the modification on glassy carbon electrode in order to overcome these problems during the electro-oxidation process of VB6.

In this work, a certain percentage of $\mathrm{GO}$ and LDH are complexed by hydrothermal method, prepared an electrochemical sensor based on $\mathrm{NiAl-LDH/GO}$ on glass carbon electrode. The NiAl-LDH/GO was extensively characterized by XRD and SEM. In order to study electronic properties of the electrochemical sensors prepared by $\mathrm{NiAl-LDH/GO}$ for electrochemical application. The obtained NiAl-LDH/GO was modified on a glassy carbon electrode by dropping method, and sensitive applied in $\mathrm{NaOH}$ solution detected VB6.

\section{Characterization of NiAl-LDH/GO}

In order to observe the surface morphology and structure of the NiAl-LDH/GO, the SEM characterization was used. Fig. 1A shows the SEM of the NiAl-LDH/GO. On the surface of the $\mathrm{GO}$, there are NiAl-LDH nano-materials wrapped and covered evenly. The results show that LDH is successfully grown on the surface of $\mathrm{GO}$, so that a new electrochemical sensor can be prepared. The XRD pattern of the NiAI-LDH/GO shows in fig. 1B, proved that we synthesized the $\mathrm{NiAl-LDH/GO}$ composite material. The Nyquist plots of bare GCE, NiAl-LDH/GCE and NiAl$\mathrm{LDH} / \mathrm{GO} / \mathrm{GCE}$ in $0.1 \mathrm{M} \mathrm{KCl}$ solution containing the redox probe $\mathrm{Fe}\left[(\mathrm{CN})_{6}\right]^{4-13-}$ showed in fig. $1 \mathrm{C}$. Compared to bare GCE, the semicircles diameter of NiAl-LDH/GCE and NiAlLDH/GO/GCE are significantly increased. However, the Ret of NiAl-LDH/GO/GCE is smaller than the NiAl-LDH/GCE, indicating between the electrode surface and the redox probe has a faster electron transfer and a lower resistance in NiAI-LDH/GO/GCE. 

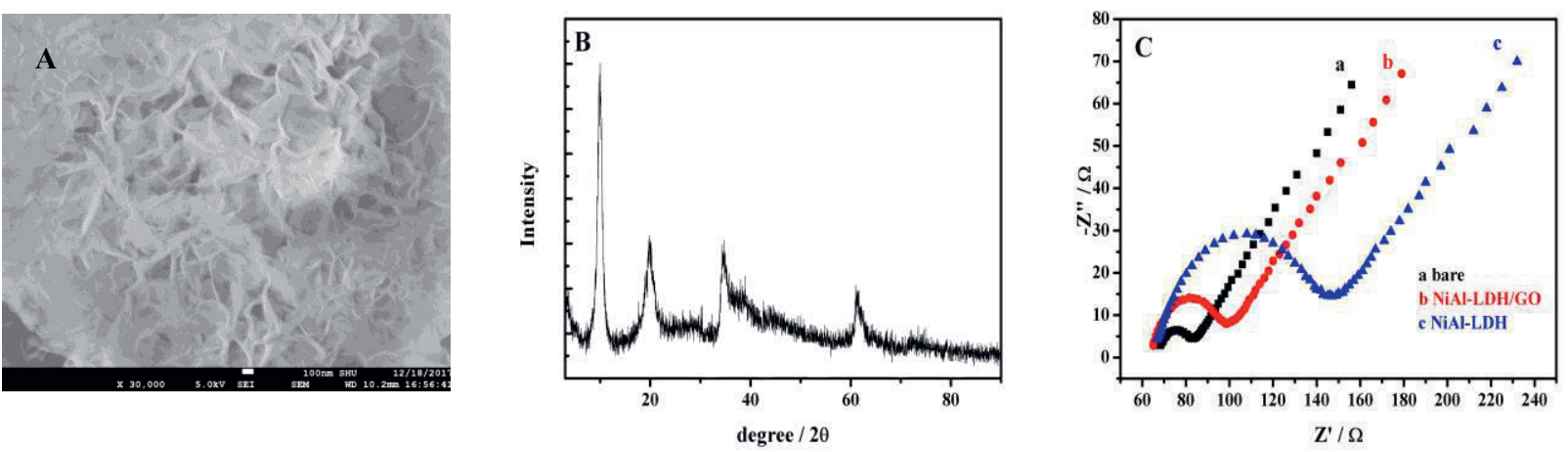

Fig. 1 A SEM of NiAI-LDH/GO; B XRD pattern of NiAl-LDH/GO; C EIS of NiAI-LDH/GO

\section{Experimental result}

For exploring the electrochemical response of the bare GCE and the modified electrode to the oxidation of VB6, we used the electrochemical methods of I-T to qualitatively investigate in a $0.15 \mathrm{M} \mathrm{NaOH}$ solution. The I-T curves for the oxidation of VB6 by bare electrode and NiAlLDH/GO modified electrode are shown in Fig. $2 \mathrm{~A}$. In the presence of $10 \mu \mathrm{M}$ VB6 as electrochemically active substances, the current response for the bare GCE is very small. Under the same conditions, there is a obvious increase of the modified electrode of NiAlLDH/GO after adding10 $\mu M$ VB6. The result shows that the electrocatalytic activity of NiAl-
LDH/GO. Under the optimal conditions, I-T detection was implemented for VB6 at NiAl$\mathrm{LDH} / \mathrm{GO} / \mathrm{GCE}$. The current response of the $\mathrm{NiAl-LDH/GO/GCE}$ to VB6 with different concentration displayed in fig. 2B. As shown in fig. $2 \mathrm{C}$, there is linear relationship between the gradually addition of $0.08-500 \mu \mathrm{M}$ VB6 and the current response of the modified electrode in the $\mathrm{NaOH}$ solution. Ip $(\mu \mathrm{A})=0.4994+$ $0.00888 \mathrm{C}(\mu \mathrm{M})\left(R^{2}=0.998\right)$ was the linear regression equation. The detection limit was estimated as $0.027 \mu \mathrm{M}(\mathrm{S} / \mathrm{N}=3)$. A good work linear range and an excellent detection limit are indicated in this work.
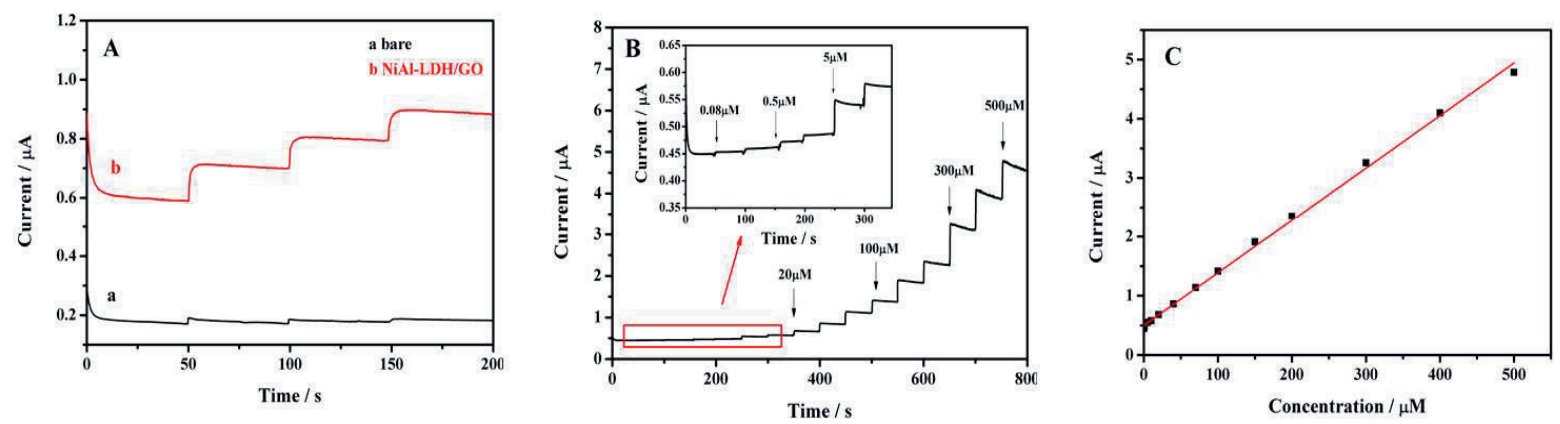

Fig. 2 A I-T of $10 \mu M$ VB6 on bare GCE and NiAl-LDH/GO/GCE; B I-T of VB6 in the concentration range from 0.08 $\mu \mathrm{M}$ to $500 \mu \mathrm{M}$ on NiAl-LDH/GO/GCE; C the linear regression of VB6 on NiAl-LDH/GO/GCE.

This work is supported by the National Natural Science Foundation of China (No. 21671132)

\section{References}

[1] J.L. Anders, B. Rasmus, K.N. Maja, et al. Fluorescence Spectroscopy as A Potential Metabonomic Tool for Early Detection of Colorectal Cancer. Metabolomics 8, 111-121 (2012); doi: 10.1007/s11306-011-0321-4.

[2] T. Nie, J.K. Xu, L.M. Lu, et al. Electroactive Species-doped Poly(3,4-ethylenedioxythiophene) Films: Enhanced Sensitivity for Electrochemical Simultaneous Determination of Vitamins B2, B6 and C. Biosensors and Bioelectronics 50, 244250 (2013); doi:10.1016/j.bios.2013.06.057.

[3] P.P. Zhang, S.Suidasari, T. Hasegawa, Vitamin B? Activates p53 and Elevates p21 Gene Expression in Cancer Cells and The Mouse Colon. Oncology Reports 31, 2371-2376 (2014); doi:10.3892/or.2014.3073.

[4] T. Saekar, S. Banerjee, A. Hussain, Significant Photocytotoxic Effect of An Iron(III) Complex of A Schiff Base Ligand Derived from Vitamin B6 and Thiosemicarbazide in Visible Light. RSC Advances 5, 29276 - 29284 (2015); doi:10.1039/c5ra04207k.. 